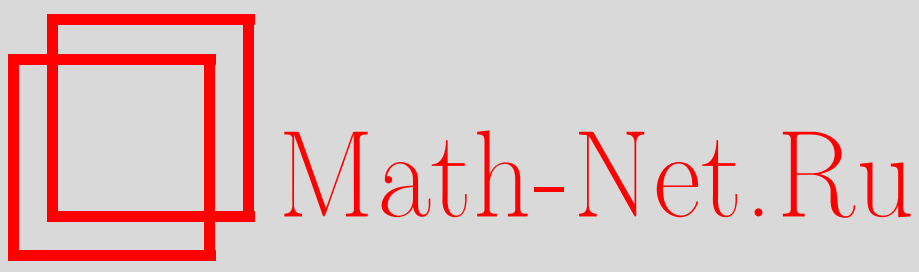

А. Е. Шишков, А. Г. Щелков, Динамика носителей энергетических решений смешанных задач для квазилинейных параболических уравнений произвольного порядка, Изв. РАН. Сер. матем., 1998, том 62, выпуск 3, 175-200

DOI: https://doi.org/10.4213/im200

Использование Общероссийского математического портала Math-Net.Ru подразумевает, что вы прочитали и согласны с пользовательским соглашением

http://www. mathnet.ru/rus/agreement

Параметры загрузки:

IP : 35.173 .219 .149

26 апреля 2023 г., 12:52:57 


\author{
А. Е. Шишков, А. Г. Щелков
}

\title{
Динамика носителей энергетических решений смешанных задач для квазилинейных параболических уравнений произвольного порядка
}

\begin{abstract}
Изучается геометрия носителей решений задачи Коши-Дирихле для широкого класса квазилинейных вырождающихся параболических уравнений произвольного порядка, модельным представителем которых является уравнение нестационарной фильтрации с нелинейным поглощением:
\end{abstract}

$$
\frac{\partial}{\partial t}\left(|u|^{q-1} u\right)-\sum_{i=1}^{n} \frac{\partial}{\partial x_{i}}\left(\left|D_{x} u\right|^{p-1} \frac{\partial u}{\partial x_{i}}\right)+b_{0}|u|^{\lambda-1} u=0, \quad b_{0}>0, \quad n \geqslant 1 .
$$

В случае $0<\lambda<p \leqslant q$ и $0<\lambda<q<p$, соответствующих "быстрой" и "медленной" диффузиям, найдены в определенном смысле точные условия на поведение начальной функции $u_{0}(x) \in L_{q+1}(\Omega)$ в окрестности границы ее носителя, обеспечивающие появление эффекта конечной и бесконечной инерции носителя произвольного энергетического решения. Установлено условие возникновения обратного движения фронта границы носителя.

Библиография: 24 наименования.

\section{$\S 1$. Введение}

В настоящей работе изучаются локализационные свойства и распространение носителей обобшенных решений следуюшей смешанной задачи Коши-Дирихле:

$$
\begin{gathered}
\frac{\partial}{\partial t}\left(|u|^{q-1} u\right)+A u+b(x, t, u)=0 \\
\forall(x, t) \in Q=\Omega \times\left(0, T_{1}\right), \quad T_{1}<\infty, \quad q>0, \\
\left.D_{x}^{\alpha} u\right|_{\Gamma=\partial \Omega \times\left(0, T_{1}\right)}=0 \quad \forall \alpha:|\alpha| \leqslant m-1, \quad m \geqslant 1, \\
u(x, 0)=u_{0}(x) \in L_{1+q}(\Omega) .
\end{gathered}
$$

Здесь $\Omega$-произвольная (возможно, неограниченная) область в $\mathbb{R}^{n}, n \geqslant 1$;

$$
A u \equiv(-1)^{m} \sum_{|\alpha|=m} D_{x}^{\alpha} a_{\alpha}\left(x, t, u, D_{x} u, \ldots, D_{x}^{m} u\right)
$$

Первому автору этой работы был выдан грант Международного научного фонда № U97000. 
причем каратеодориевы функции $a_{\alpha}(x, t, \xi), b(x, t, s)$ удовлетворяют условиям

$$
\begin{gathered}
\sum_{|\alpha|=m} a_{\alpha}(x, t, \xi) \xi_{\alpha} \geqslant d_{0} \sum_{|\beta|=m}\left|\xi_{\beta}\right|^{p+1} \quad \forall(x, t, \xi) \in Q \times \mathbb{R}^{N(m)}, \quad d_{0}>0 \\
a_{\alpha}|x, t, \xi| \leqslant d_{1} \sum_{|\beta|=m}\left|\xi_{\beta}\right|^{p}, \quad d_{1}<\infty, \quad p>0 \\
d_{2}^{\prime}|s|^{1+\lambda}>b(x, t, s) s \geqslant d_{2}|s|^{1+\lambda} \quad \forall(x, t, s) \in Q \times \mathbb{R}^{1} \\
d_{2}^{\prime}<\infty, \quad d_{2}>0, \quad \lambda>0
\end{gathered}
$$

через $N(m)$ обозначено число различных $n$-мерных мультииндексов длины, не большей, чем $m$.

Класс уравнений, определяемый выражением (1.1), достаточно широк и охватьвает большое количество уравнений математической физики. Например, в него входит уравнение нестационарной ньютоновской фильтрации с нелинейной абсорбцией

$$
\frac{\partial}{\partial t}\left(|u|^{q-1} u\right)-\triangle u+|u|^{\lambda-1} u=0, \quad u=u(x, t), \quad(x, t) \in \mathbb{R}^{n} \times(0, T) .
$$

Для этого уравнения в случае $n=1$ Л.К. Мартинсоном, К.Б. Павловым [1] и А.С. Калашниковым [2], [3] было установлено при $0<\lambda<q<1$ свойство локализации решений и конечность скорости изменения носителей решений задачи Коши. Для $n>1$ эти результаты обобщались в работах [4], [5]. Многие авторы устанавливали оценки скорости изменения носителей. С другой стороны, были обнаружены ситуации, когда носитель решений может изменяться более сложным образом. Так, Р. Кершнером [6], [7] найдены решения, носитель которых строго уменьшается с течением времени. Были также установлены условия, при которых решение задачи Коши со строго положительной начальной функцией $u_{0}(x)$ с течением времени обрашается в нуль в некоторых подобластях (так называемые "мертвые зоны") и даже обрашается в тождественный нуль за конечное время(см. [2], [8]-[13]). Отметим, что во всех перечисленных работах использовалась техника, связанная с построением различных суб- и суперрешений и поэтому, естественно, не пригодная для общих уравнений. В ряде работ локализационные свойства обобшенных решений уравнений второго порядка структуры (1.1) изучались методом так назьваемых локальных энергетических оценок (например, см. [14]- [18]). Конечность скорости изменения носителей обобщенных решений квазилинейных уравнений структуры (1.1) произвольного порядка и некоторые ее оценки были установлены $\Phi$. Бернисом [19]. При этом существенно использовались доказанные в [19] весовые интерполящионные неравенства типа Ниренберга-Гальярдо. Другой способ, основанный на априорных оценках типа оценок, которые выражают принцип Сен-Венана в теории упругости, был предложен в работах [20], [21]. С помощью этого метода в настоящей работе устанавливаются для произвольных энергетических обобшенных решений задачи (1.1)-(1.3) условия конечной и бесконечной задержки расширения носителя (конечная и бесконечная инерция). Выводятся условия на поведение интегральной нормы начальной функции в окрестности ее носителя, обеспечивающие сжатие носителя для всех $t>0$, и даны оценки скорости сжатия. 


\section{§ 2. Формулировки основных результатов}

Введем функциональное пространство

$$
W \equiv W_{p+1}^{m}(\Omega, \partial \Omega) \cap L_{q+1}(\Omega) .
$$

Через $W_{\beta}^{m}(\Omega, R), R \subset \partial \Omega$, обозначается, как обычно, замыкание в норме $W_{\beta}^{m}(\Omega)$ множества функций из $C^{\infty}(\bar{\Omega})$, обрашающихся в нуль в окрестности $R$.

ОПРЕДЕЛЕНИЕ. Энергетическим обобщенным решением задачи (1.1)-(1.3) называем функцию

$$
u(x, t) \in C\left(\left[0, T_{1}\right] ; L_{q+1}(\Omega)\right) \cap L_{p+1}(0, T ; W) \cap L_{1+\lambda}\left(\Omega \times\left(0, T_{1}\right)\right),
$$

удовлетворяющую уравнению (1.1) в смысле распределений из $D^{\prime}(Q)$ и начальному условию (1.3).

ЗАмЕчАниЕ 1. Следуя [19], из определения выводится формула интегрирования по частям:

$$
\begin{aligned}
\frac{\varphi(T) q}{q+1} & \int_{\Omega}|u(x, T)|^{q+1} \eta(x) d x \\
& +\int_{\Omega \times(0, T)}\left[\sum_{|\alpha|=m} a_{\alpha}\left(x, t, u, D_{x} u, \ldots, D_{x}^{m} u\right) \varphi\right. \\
& \times D_{x}^{\alpha}(\eta u)+b(x, t, u(x, t)) u(x, t) \eta(x) \varphi(t) \\
& \left.-\frac{q}{q+1}|u(x, t)|^{q+1} \varphi_{t}^{\prime}(t) \eta(x)\right] d x d t \\
= & \frac{q}{q+1} \varphi(0) \int_{\Omega}\left|u_{0}(x)\right|^{q+1} \eta(x) d x \quad \forall T: 0<T \leqslant T_{1},
\end{aligned}
$$

где $\varphi(t)$ и $\eta(x)$ - произвольные функции соответственно из $C^{1}\left[0, T_{1}\right]$ и $W_{\infty}^{m}(\Omega)$.

ЗАмЕчАниЕ 2 . При $q=1$ разрешимость задачи (1.1)-(1.3) достаточно хорошо изучена (см. [22]). В случае $q \neq 1$ существование энергетического обобщенного решения при $b(x, t, s)=|s|^{\lambda-1} s$ и вариационной структуре оператора $A$ установлено в [19].

Введем теперь следующие обозначения. Если $\Omega \subset \mathbb{R}^{n}, u_{0}(x)$ - область и начальная функция из (1.1)-(1.3), то $\Omega(s)=\Omega \cap\left\{x=\left(x^{\prime}, x_{n}\right) \in \mathbb{R}^{n}: x_{n}<s\right\}$,

$$
\begin{aligned}
Q(s) & =\Omega(s) \times\left(0, T_{1}\right) \quad \forall s \in \mathbb{R}^{1} \\
f(s) & \equiv \int_{\Omega(s)}\left|u_{0}(x)\right|^{q+1} d x .
\end{aligned}
$$

На протяжении всей работы считаем, что при некотором $s_{0}>-\infty$

$$
\operatorname{supp} u_{0}(x) \cap \Omega\left(s_{0}\right)=\varnothing \Longrightarrow f(s)=0 \quad \forall s \leqslant s_{0} .
$$


ТЕОРЕМА 1 (эффект конечной инерции носителя решения при медленной диффузии). Пусть в уравнении (1.1)

$$
0<q<p
$$

Пусть $u_{0}(x)$, кроме условия (2.2), удовлетворяет также следующему условию малости в окрестности $\partial \Omega\left(s_{0}\right)$ :

$$
f(s) \leqslant k_{1}\left(s-s_{0}\right)^{\alpha_{1}} \quad \forall s>s_{0}, \quad k_{1}<\infty, \quad \alpha_{1}=n+\frac{m(p+1)(q+1)}{p-q} .
$$

Тогда существует $\tau=\tau\left(k_{1}\right)>0$ такое, что для любого энергетического решения $и(x, t)$ задачи (1.1)-(1.3)

$$
\operatorname{supp}_{x} u(x, t) \cap \Omega\left(s_{0}\right)=\varnothing \quad \forall t: 0<t<\tau
$$

ТЕОРЕма 2 (бесконечная инерция носителя при медленной диффузии). Пусть в уравнении (1.1)

$$
0<\lambda<q<p
$$

и $u_{0}(x)$, кроме условия (2.2), удовлетворяет следующему условию малости:

$$
f(s) \leqslant k_{2}\left(s-s_{0}\right)^{\alpha_{2}} \quad \forall s>s_{0}, \quad k_{2}<\infty, \quad \alpha_{2}=n+\frac{m(p+1)(q+1)}{p-\lambda} .
$$

Тогда существует постоянная $k_{2}^{(0)}>0$ такая, что для любого энергетического решения $u(x, t)$ задачи (1.1)-(1.3)

$$
\operatorname{supp}_{x} u(x, t) \cap \Omega\left(s_{0}\right)=\varnothing \quad \forall t>0,
$$

как только постоянная $k_{2}$ из условия (2.7) удовлетворяет неравенству $k_{2}<k_{2}^{(0)}$.

ЗАМЕчАНИЕ 3. Эффект конечной инерции для энергетических решений уравнений второго порядка $(m=1)$ типа медленной диффузии с сильной абсорбцией (условие (2.6)) изучался в работах [16]-[18], причем в последней из этих работ методом, не допускаюшим прямого обобщения на уравнения высокого порядка при $0<\lambda<q<p$ и условии малости на $u_{0}(x)$, которое после дополнительных преобразований может быть записано в виде

$$
\int_{0}^{c} s^{-1-\frac{\alpha_{2}(p+1)}{p}} f\left(\left(s+s_{0}\right)\right)^{\frac{p+1}{p}} d s<\infty \quad \forall c>0
$$

Заметим, что условие $\left(2.7^{*}\right)$ является более жестким, чем условие (2.7). Функция $f_{0}(s)=k\left(s-s_{0}\right)^{\alpha_{2}}, k<k_{2}^{(0)}$, удовлетворяет условию $(2.7)$, но не удовлетворяет $\left(2.7^{*}\right)$. Отметим еше, что в работе [21] показано появление эффекта бесконечной инерции энергетических решений уравнений произвольного порядка вида (1.1) при $q=1$ и выполнении для начальной функции условия малости (2.7). 
ТЕОРЕмА 3 (бесконечная инерция носителя при быстрой диффузии). Пусть в уравнении (1.1)

$$
0<\lambda<p \leqslant q, \quad \frac{n q-m(q+1)}{n+m(q+1)} \leqslant p,
$$

и $u_{0}(x)$, кроме условия (2.2), удовлетворяет следующему условию малости:

$$
f(s) \leqslant k_{3}\left(s-s_{0}\right)^{\alpha_{2}} \quad \forall s>s_{0}, \quad k_{3}<\infty, \quad \alpha_{2}=n+\frac{m(p+1)(q+1)}{p-\lambda} .
$$

Тогда существует постоянная $k_{3}^{(0)}: 0<k_{3}^{(0)}<k_{2}^{(0)}$, такая, что для любого әнергетического решения $и(x, t)$ задачи (1.1), (1.2) имеет место свойство (2.8) в случае, если $k_{3}<k_{3}^{(0)}$.

TЕОРема 4 (обратное движение фронта носителя решения при медленной диффузии). В условиях теоремы 2 существует такая постоянная $k_{2}^{(1)}: 0<$ $k_{2}^{(1)}<k_{2}^{(0)}$, что при удовлетворении начальной функиией $u_{0}(x)$ условия малости $(2.7)$ с произвольным $k_{2}<k_{2}^{(1)}$ обязательно существует $\delta=\delta\left(k_{2}\right)>0$ maкоe, чmo

$$
\operatorname{supp}_{x} u(x, t) \cap \Omega(l(t))=\varnothing \quad \forall t>0,
$$

где $и(x, t)$ - произвольное әнергетическое решение задачи (1.1)-(1.3),

$$
l(t)=s_{0}+\delta t^{\frac{p-\lambda}{m(p+1)(q-\lambda)}} .
$$

Пусть начальная функиия удовлетворяет более сильному условию малости, чем (2.7);

$$
f(s) \leqslant f_{1}(s)<f_{0}(s) \equiv k_{2}^{(1)}\left(s-s_{0}\right)^{\alpha_{2}} \quad \forall s>s_{0} .
$$

Тогда существуют постоянные $D<\infty u 0<d<1$, зависящие только от известных параметров уравнения (1.1), такие, что при выполнении для мажсоранты $f_{1}(s)$ условия правильной монотонности:

$$
f_{1}\left(s_{0}+s-D\left(f_{1}\left(s_{0}+s\right)\right)^{\frac{1}{\alpha_{2}}}\right) \geqslant d \cdot f_{1}\left(s_{0}+s\right) \quad \forall s>0
$$

свойство (2.11) имеет место $c$

$$
l(t)=s_{0}+c_{1}\left[f_{1}^{-1}\left(C_{1} t^{\frac{n(p-\lambda)+m(p+1)(q+1)}{m(p+1)(q-\lambda)}}\right)-s_{0}\right], \quad c_{1}>0, \quad 0<C_{1}<\infty .
$$

Здесь $f_{1}^{-1}-$ функция, обратная $\kappa f_{1}(s)$.

СлЕДСТвиЕ 1. Если выполняется следующее соотношение на параметры уравнения (1.1):

$$
\frac{p-\lambda}{m(p+1)(q-\lambda)} \leqslant 1 \Longleftrightarrow \lambda \leqslant \frac{p+m(p+1) q}{m(p+1)-1},
$$

то, как следует из (2.12), стартовая скорость движсения фронта отлична от нуля. 
ТЕОРЕма 5 (обратное движение фронта носителя при быстрой диффузии). $B$ условиях теоремы 3 существует постоянная $k_{3}^{(1)}: 0<k_{3}^{(1)}<k_{3}^{(0)}$, такая, что если $u_{0}(x)$ удовлетворяет ограничению $(2.10)$ с произвольной $k_{3}<k_{3}^{(1)}$, то соответствующее энергетическое решение задачи (1.1)-(1.3) обладает свойством (2.11) с

$$
l(t)=s_{0}+\delta_{1} t^{\frac{p-\lambda}{m(p+1)(q-\lambda)}} \quad \forall t>0
$$

əде $\delta_{1}=\delta_{1}\left(k_{3}\right)>0$.

ЗАмЕчАниЕ 4 . В случае, если начальная функция $u_{0}(x)$ удовлетворяет более сильному условию малости, чем (2.10):

$$
f(s) \leqslant f_{1}(s)<f_{0}(s) \equiv k_{3}^{(1)}\left(s-s_{0}\right)^{\alpha_{2}} \quad \forall s>s_{0},
$$

то так же, как и в теореме 4, можно установить более быстрое смещение фронта носителя, чем то, что определяется функцией $l(t)$ из (2.14).

\section{§ 3. Конечная и бесконечная инерции решений}

Доказательство основных утверждений разобьем на ряд лемм, некоторые из которых являются общими для нескольких теорем. Введем еще семейства подобластей:

$$
K(s, \delta)=\Omega(s) \backslash \Omega(s-\delta), \quad G(s, \delta)=Q(s) \backslash Q(s-\delta) \quad \forall s, \delta \in \mathbb{R}^{1} .
$$

Мы систематически будем использовать интерполящионное неравенство Ниренберга-Гальярдо [24], которое в случае интересующего нас семейства подобластей $K(s, \delta)$ принимает вид

$$
\begin{gathered}
\left\|D_{x}^{j} v\right\|_{\alpha, K(s, \delta)} \leqslant d_{4} \delta^{-j-\frac{n(\alpha-\gamma)}{\alpha \gamma}}\|v\|_{\gamma, K(s, \delta)}+d_{3}\left\|D_{x}^{m} v\right\|_{\beta, K(s, \delta)}^{\theta}\|v\|_{\gamma, K(s, \delta)}^{1-\theta} \\
d_{3}<\infty, \quad d_{4}<\infty, \quad 0 \leqslant j<m .
\end{gathered}
$$

Здесь $v(x)$ - произвольная функция из соболевского пространства

$$
\breve{W}_{\beta}^{m}(K(s, \delta), \Omega \cap \partial K(s, \delta)) \cap L_{\gamma}(K(s, \delta)), \quad\|v\|_{\alpha, B}^{\alpha} \equiv \int_{B}|v|^{\alpha} d x
$$

$\gamma>0, \alpha>1, \beta>1 ;$ постоянная $\theta \in\left[\frac{j}{m}, 1\right]$ такова, что

$$
\frac{1}{\alpha}-\frac{j}{n}=\left(\frac{1}{\beta}-\frac{m}{n}\right) \theta+(1-\theta) \frac{1}{\gamma}
$$

постоянные $d_{3}, d_{4}$ зависят только от параметров $m, n$ и не зависят от геометрии области $\Omega$, от $\alpha, \beta, \gamma, s, \delta$ и от функции $v(x)$. 
ЛЕмма 1. Пусть и( $x, t)$ - произвольное әнергетическое обобщенное решение задачи (1.1)-(1.3). Тогда для произвольных $0<\delta<s, 0<T<T_{1}$ имеет место следующее энергетическое неравенство:

$$
\begin{gathered}
\int_{\Omega(s-\delta)}|u(x, T)|^{q+1} d x+\int_{Q^{(T)}(s-\delta)}\left(T^{-1}|u|^{q+1}+\left|D_{x}^{m} u\right|^{p+1}+|u|^{\lambda+1}\right) \varphi_{T}(t) d x d t \\
\leqslant c\left(\delta^{-m(p+1)} \int_{G^{(T)}(s, \delta)}|u|^{p+1} \varphi_{T}(t) d x d t+f(s)\right) \equiv J_{T}(s, \delta) \\
\varphi_{T}(t) \equiv \exp \left(-\frac{t}{T}\right) .
\end{gathered}
$$

Здесь и в дальнейшем через $c$ обозначаются различные постоянные, зависяшие лишь от известных параметров уравнения $(1.1), Q^{(T)}(s)=\Omega(s) \times(0, T)$, $G^{(T)}(s, \delta)=Q^{(T)}(s) \backslash Q^{(T)}(s-\delta)$.

ДокАЗАТЕЛЬСТво. Введем срезающую функцию $\eta(h) \in C^{m}\left(\mathbb{R}_{+}^{1}\right)$ такую, что $\eta(h)=0$ при $h>s, \eta(h)=1$ при $h<s-\delta$ и

$$
\left|D^{j} \eta\right| \leqslant d_{5} \cdot \delta^{-j}, \quad j=1, \ldots, m, \quad d_{5}<\infty .
$$

Подставим в интегральное тождество $(2.1) \varphi(t) \equiv \varphi_{T}(t)$ и в качестве $\eta$ срезаюшую функцию $\eta\left(x_{n}\right)$. Используем для оценки сверху слагаемого

$$
\int_{G^{(T)}(s, \delta)} a_{\alpha}\left(x, t, \ldots, D_{x}^{m} u\right) D_{x}^{\beta} u D^{\alpha-\beta} \eta \varphi_{T}(t) d x d t, \quad|\beta|=i, \quad i=0, \ldots, m-1,
$$

неравенство Гёльдера, оценку (1.5), интерполяционное неравенство (3.1) при $j=i$, $\alpha=\beta=\gamma=p+1$, условие (1.6) и, наконец, неравенство Юнга с " $\varepsilon$ ". Собирая все эти оценки, приходим к неравенству

$$
\begin{aligned}
& \int_{\Omega(s)}|u(x, T)|^{q+1} \eta d x+\int_{Q^{(T)}(s)}\left(T^{-1}|u|^{q+1}+\left|D_{x}^{m} u\right|^{p+1}+|u|^{\lambda+1}\right) \varphi_{T}(t) \eta d x d t \\
& \leqslant \varepsilon \int_{G^{(T)}(s, \delta)}\left|D_{x}^{m} u\right|^{p+1} \varphi_{T} d x d t+\frac{c_{1}(\varepsilon)}{\delta^{m(p+1)}} \\
& \times \int_{G^{(T)}(s, \delta)}|u|^{p+1} \varphi_{T} d x d t+c_{2} f(s) \quad \forall \varepsilon>0
\end{aligned}
$$

Введем функции

$$
\begin{aligned}
A(s) & \equiv \int_{\Omega(s)}|u(x, T)|^{q+1} d x+\int_{Q^{(T)}(s)}\left(T^{-1}|u|^{q+1}+|u|^{\lambda+1}\right) \varphi_{T}(t) d x d t \\
B(s) & \equiv \int_{Q^{(T)}(s)}|u|^{p+1} \varphi_{T}(t) d x d t \\
H(s) & \equiv \int_{Q^{(T)}(s)}\left|D_{x}^{m} u\right|^{p+1} \varphi_{T}(t) d x d t .
\end{aligned}
$$

Можем переписать соотношение (3.4) в виде

$$
A(s-\delta)+H(s-\delta) \leqslant \varepsilon H(s)+c_{1}(\varepsilon) \delta^{-m(p+1)} B(s)+c_{2} f(s) .
$$


Так как в (3.5) $s>\delta>0$ могут принимать любые значения, то справедливы и следующие неравенства:

$$
\begin{aligned}
A\left(s-\frac{\delta}{2^{j-1}}\right) & +H\left(s-\frac{\delta}{2^{j-1}}\right) \leqslant \varepsilon H\left(s-\frac{\delta}{2^{j}}\right) \\
& +\left(\frac{2^{j}}{\delta}\right)^{m(p+1)} c_{1}(\varepsilon) B(s)+c_{2} f(s) \quad \forall j \in \mathbb{N} .
\end{aligned}
$$

Здесь мы воспользовались монотонностью функций $B(s)$ и $f(s)$. Стартуем с неравенства (3.6) при $j=1$, затем член $H\left(s-\frac{\delta}{2}\right)$ в правой части получившегося неравенства оцениваем при помоши (3.6) с $j=2$, далее продолжаем циклически $i$ раз. Собирая все слагаемые, приходим к неравенству

$$
\begin{aligned}
A(s-\delta)+H(s-\delta) \leqslant & \varepsilon^{i} H\left(s-\frac{\delta}{2^{i}}\right)+\left(\frac{2}{\delta}\right)^{m(p+1)} c_{1}(\varepsilon) B(s) \\
& \times \sum_{k=0}^{i-1}\left(\varepsilon 2^{m(p+1)}\right)^{k}+c_{2} f(s) \sum_{k=0}^{i-1} \varepsilon^{k} \quad \forall i \in \mathbb{N} .
\end{aligned}
$$

Полагая в последнем неравенстве $\varepsilon=2^{-1-m(p+1)}$ и устремляя $i \mathrm{~K} \infty$, получаем

$$
A(s-\delta)+H(s-\delta) \leqslant 2\left(\frac{2}{\delta}\right)^{m(p+1)} c_{1}\left(2^{-1-m(p+1)}\right) B(s)+\frac{2^{m(p+1)+1} f(s)}{2^{m(p+1)+1}-1} c_{2}
$$

что совпадает с требуемой оценкой (3.2).

ЛЕмма 2. Пусть $и(x, t)$ - произвольное әнергетическое решение задачи (1.1)-(1.3). Введем следующие функиии, связанные с этим решением:

$$
\begin{aligned}
I_{T}(s) & =\int_{Q^{(T)}(s)}|u|^{p+1} \varphi_{T}(t) d x d t \\
\Phi_{T}(s, h) & =\int_{0}^{T}\left(\int_{\Omega(s)}|u|^{q+1} \eta\left(x_{n}\right) d x\right)^{h} \varphi_{T}(t) d x d t
\end{aligned}
$$

əде $0<h, \quad 0<T \leqslant T_{1} ; \varphi_{T}(t)$ и $\eta\left(x_{n}\right)$ из леммы 1.

Тогда имеет место следующее соотношение:

$$
\begin{gathered}
I_{T}(s-\delta) \leqslant c J_{T}(s, \delta)^{\theta}\left(J_{T}(s+\delta, \delta)^{\frac{p+1}{q+1}-h} \Phi_{T}(s, h)\right)^{1-\theta} \\
\forall h: 0<h \leqslant \frac{p+1}{q+1}, \quad \forall \delta<s \\
\theta=\frac{n(p-q)}{n(p-q)+m(1+q)(1+p)}
\end{gathered}
$$


ДоКАЗАТЕЛЬСТво. Интерполящионное неравенство (3.1) при $\delta \rightarrow \infty, j=0$, $\alpha=p+1, \beta=p+1, \gamma=1+q$ дает

$$
\begin{aligned}
\int_{\Omega(s)} & |u(x, t)|^{p+1} d x \\
& \leqslant d_{3}^{p+1}\left(\int_{\Omega(s)}\left|D_{x}^{m} u(x, t)\right|^{p+1} d x\right)^{\theta}\left(\int_{\Omega(s)}|u|^{q+1} d x\right)^{\frac{(1-\theta)(p+1)}{(q+1)}} \quad \forall s \in \mathbb{R}^{1},
\end{aligned}
$$

где $\theta$ из (3.7). Умножая это неравенство на $\varphi_{T}(t)$ и интегрируя его по $t$, получим, применяя неравенство Гёльдера,

$$
\begin{aligned}
I_{T}(s) \leqslant & d_{3}^{p+1}\left(\int_{Q^{(T)}(s)}\left|D^{m} u\right|^{p+1} \varphi_{T} d x d t\right)^{\theta} \\
& \times\left(\int_{0}^{T}\left(\int_{\Omega(s)}|u|^{q+1} d x\right)^{\frac{p+1}{q+1}} \varphi_{T} d t\right)^{1-\theta} \quad \forall s \in \mathbb{R}^{1} .
\end{aligned}
$$

Отсюда, с учетом оценки (3.2), выводим

$$
I_{T}(s-\delta) \leqslant c J_{T}(s, \delta)^{\theta}\left(\Psi_{T}\left(s-\delta, \frac{p+1}{q+1}\right)\right)^{1-\theta} \quad \forall s, \delta \in \mathbb{R}^{1},
$$

где $\Psi_{T}(s, h) \equiv \int_{0}^{T}\left[\int_{\Omega(s)}|u|^{q+1} d x\right]^{h} \varphi_{T}(t) d t$.

Оценим отдельно второй сомножитель в (3.8) сверху. Для этого введем функцию

$$
\zeta^{(h)}(t)=\int_{0}^{t}\left(\int_{\Omega(s)}|u(x, \theta)|^{q+1} \eta d x\right)^{h} \varphi_{T}(\theta) d \theta .
$$

Легко проверить справедливость следующего равенства:

$$
\int_{Q^{(T)}(s)}|u(x, t)|^{q+1} \eta\left(x_{n}\right) \frac{d}{d t}\left(\zeta^{(h)}(t)\right) d x d t=\zeta^{(h+1)}(T) \quad \forall h>0 .
$$

Поэтому, подставляя в интегральное тождество $(2.1)$ в качестве $\varphi(t)$ введенную функцию $\zeta^{(h)}(t)$, получим, используя условия (1.5), (1.6), неравенство

$$
\begin{aligned}
\zeta^{(h+1)}(T) \equiv & \Phi_{T}(s, h+1)=\zeta^{(h)}(T) \int_{\Omega(s)}|u(x, T)|^{q+1} \eta d x \\
& +\int_{Q^{(T)}(s)}\left[\sum_{|\alpha|=m} a_{\alpha}\left(x, \ldots, D^{m} u\right) D^{\alpha}(u \eta)+b(x, t, u) u \eta\right] \zeta^{(h)}(t) d x d t \\
\leqslant & c \zeta^{(h)}(T)\left[\int_{\Omega(s)}|u(x, T)|^{q+1} \eta d x+\int_{Q^{(T)}(s)}\left(\left|D_{x}^{m} u\right|^{p+1}+|u|^{\lambda+1}\right) \eta d x d t\right. \\
& \left.+\int_{G^{(T)}(s, \delta)}\left|D_{x}^{m} u\right|^{p} \sum_{i=1}^{m} \delta^{-i}\left|D_{x}^{m-i} u\right| d x d t\right] .
\end{aligned}
$$


Применяя для оценки сверху последнего слагаемого в квадратных скобках неравенство Гёльдера, затем интерполяционное неравенство (3.1) при $j=m-i$, $\alpha=\beta=\gamma=p+1$ и далее используя оценку (3.2) с $s+\delta$ вместо $s$, получим

$$
\Phi_{T}(s, h+1) \leqslant c \Phi_{T}(s, h) J_{T}(s+\delta, \delta) \quad \forall h>0 .
$$

Отсюда, еще раз применяя неравенство Гёльдера, легко получаем

$$
\Phi_{T}\left(s, h_{2}\right) \leqslant c \Phi_{T}\left(s, h_{1}\right) J_{T}(s+\delta, \delta)^{h_{2}-h_{1}} \quad \forall h_{2}>h_{1}>0 .
$$

Очевидно, что

$$
\Psi_{T}(s-\delta, h) \leqslant \Phi_{T}(s, h) \quad \forall h>0 .
$$

Поэтому из оценки (3.8) с учетом соотношения (3.9) при $h_{2}=\frac{p+1}{q+1}, h_{1}=h$ получаем

$$
\begin{aligned}
I_{T}(s-\delta) & \leqslant c J_{T}(s, \delta)^{\theta}\left[\Phi_{T}\left(s, \frac{p+1}{q+1}\right)\right]^{1-\theta} \\
& \leqslant c J_{T}(s, \delta)^{\theta}\left[J_{T}(s+\delta, \delta)^{\frac{p+1}{q+1}-h} \Phi_{T}(s, h)\right]^{1-\theta}
\end{aligned}
$$

что и доказывает лемму 2.

ДОКАЗАТЕЛЬСТВО ТЕОРЕМЫ 1. Заметим, что в силУ основной энергетической оценки (3.2)

$$
\begin{aligned}
\Phi_{T}(s, 1) & \equiv \int_{Q^{(T)}(s)}|u|^{q+1} \varphi_{T}(t) \eta\left(x_{n}\right) d x d t \\
& \leqslant \int_{Q^{(T)}(s)}|u|^{q+1} \varphi_{T} d x d t \leqslant c T J_{T}(s+\delta, \delta) .
\end{aligned}
$$

Поэтому из соотношения (3.7) при $h=1$ вытекает

$$
\begin{aligned}
I_{T}(s-\delta) & \leqslant c J_{T}(s, \delta)^{\theta} J_{T}(s+\delta, \delta)^{\frac{(p+1)(1-\theta)}{q+1}} T^{1-\theta} \\
& \leqslant c_{1} T^{1-\theta} J_{T}(s+\delta, 2 \delta)^{1+\frac{(p-q)(1-\theta)}{q+1}} .
\end{aligned}
$$

Делая очевидную замену переменных, отсюда получаем

$$
I_{T}(s-\delta) \leqslant c T^{1-\theta} J_{T}(s, \delta)^{1+\frac{(p-q)(1-\theta)}{q+1}} \quad \forall s>\delta .
$$

Вспоминая определение функции $J_{T}(s, \delta)$ и обозначая

$$
\kappa=\frac{(p-q)(1-\theta)}{(q+1)}=\frac{m(p+1)(p-q)}{n(p-q)+m(q+1)(p+1)},
$$

выводим из последнего соотношения

$$
I_{T}(s-\delta) \leqslant c_{1} T^{1-\theta} \frac{\left(\Delta I_{T}(s)\right)^{1+\kappa}}{\delta^{m(1+\kappa)(1+p)}}+c_{2} T^{1-\theta} f(s)^{1+\kappa},
$$

где $\Delta I_{T}(s)=I_{T}(s)-I_{T}(s-\delta)$. Положим в неравенстве (3.10)

$$
\delta=\delta(s, T) \equiv T^{\frac{1-\theta}{m(p+1)(1+\kappa)}} I_{T}(s)^{\frac{\kappa}{m(1+\kappa)(p+1)}} .
$$


В результате после тривиальных преобразований получим

$$
I_{T}(s-\delta(s, T)) \leqslant \nu I_{T}(s)+\nu_{1} T^{1-\theta} f(s)^{1+\kappa},
$$

где $\nu=\frac{c_{1}}{1+c_{1}}<1, \nu_{1}=\frac{c_{2}}{1+c_{1}}$. Умножим обе части последнего неравенства на $T^{\frac{1-\theta}{\kappa}}$, а затем возведем в степень $\frac{\kappa}{m(1+\kappa)(p+1)}$. В результате для функции $R_{T}(s) \equiv$ $T^{\frac{1-\theta}{m(1+\kappa)(p+1)}} I_{T}\left(s_{0}+s\right)^{\frac{\kappa}{m(1+\kappa)(p+1)}}$ получим следуюшее соотношение:

$$
R_{T}\left(s-R_{T}(s)\right) \leqslant \nu_{2} R_{T}(s)+\nu_{3} F_{T}(s) \quad \forall s \in \mathbb{R}^{1}
$$

где

$$
\nu_{2}=\nu^{\frac{\kappa}{m(1+\kappa)(p+1)}}<1, \quad \nu_{3}=\nu_{1}^{\frac{\kappa}{m(1+\kappa)(p+1)}}, \quad F_{T}(s)=T^{\frac{1-\theta}{m(p+1)}} f\left(s_{0}+s\right)^{\frac{\kappa}{m(p+1)}} .
$$

Вернемся еще раз к соотношению (3.10). Полагая в нем $\delta=2^{-1} s$ и устремляя $s$ к $\infty$, получим, учитывая ограниченность функций $I_{T}(s), f(s)$, справедливость следующей оценки:

$$
I_{T}(\infty) \leqslant c_{2} T^{1-\theta} f(\infty)^{1+\kappa}
$$

Поэтому существует (возможно, достаточно большое) число $s^{\prime}<\infty$ такое, что $I_{T}(s) \leqslant 2 c_{2} T^{1-\theta} f(\infty)^{1+\kappa} \forall s>s^{\prime}, \forall T>0$, и, следовательно,

$$
R_{T}(s) \leqslant\left(2 c_{2}\right)^{\frac{\kappa}{m(1+\kappa)(p+1)}} F_{T}(s) \quad \forall s>s^{\prime}-s_{0} .
$$

Заметим еще, что в силу условия (2.4) имеется следующая оценка для функции $F_{T}(s)$ :

$$
\begin{aligned}
F_{T}(s) & \leqslant T^{\frac{1-\theta}{m(p+1)}} k_{1}^{\frac{\kappa}{m(p+1)}} s^{\frac{[n(p-q)+m(p+1)(q+1)] \kappa}{(p-q) m(p+1)}}=k_{1}^{\frac{p-q}{N}} T^{\frac{1+q}{N}} s \\
N & =m(p+1)(q+1)+n(p-q) .
\end{aligned}
$$

Теперь к функциям $R_{T}(s), F_{T}(s)$, удовлетворяюшим соотношению (3.11) и априорным оценкам (3.12), (3.13), применим лемму 4 из дополнения. В силу этой леммы

$$
R_{T}(s)=0 \quad \forall s>0
$$

если выполнены условия (5.4):

$$
\nu_{3} k_{1}^{\frac{p-q}{N}} T^{\frac{1+q}{N}}<\left(\lambda-\nu_{2}\right)(1-\lambda)
$$

где $\left(\lambda-\nu_{2}\right)=\left(2 c_{2}\right)^{\frac{-\kappa}{m(1+\kappa)(p+1)}}=\left(2 c_{2}\right)^{-\frac{p-q}{M}}, M=m(p+1)^{2} n(p-q)$.

Следовательно, свойство (3.14) будет вьполняться до тех пор, пока вьполняется условие (3.15), т.е.

$$
T<\nu_{3}^{-\frac{N}{1+q}} k_{1}^{-\frac{p-q}{1+q}}\left(2 c_{2}\right)^{-\frac{(p-q) N}{(1+q) M}}\left(1-\nu_{2}-\left(2 c_{2}\right)^{-\frac{(p-q)}{M}}\right)^{\frac{N}{1+q}} \equiv \tau\left(k_{1}\right) .
$$

Тем самым в силу соотношения (3.14) верно с указанным значением $\tau\left(k_{1}\right)<\infty$ соотношение (2.5). Теорема 1 доказана. 
ДоКАЗАТЕЛЬСТВо ТЕОРЕМЫ 2. Запишем интерполящионное неравенство (3.1) при $j=0, \alpha=1+q, \beta=p+1, \gamma=1+\lambda$ и $\delta \rightarrow \infty$ :

$$
\begin{aligned}
\int_{\Omega(s)}|u|^{1+q} d x \leqslant & d_{3}^{q+1}\left(\int_{\Omega(s)}\left|D_{x}^{m} u\right|^{p+1} d x\right)^{\frac{\theta_{1}(1+q)}{(p+1)}} \\
& \times\left(\int_{\Omega(s)}|u|^{\lambda+1} d x\right)^{\frac{\left(1-\theta_{1}\right)(q+1)}{\lambda+1}} \quad \forall s \in \mathbb{R}^{1}, \\
\theta_{1}= & \frac{n(p+1)(q-\lambda)}{(1+q)[n(p-\lambda)+m(p+1)(1+\lambda)]} .
\end{aligned}
$$

Отсюда, применяя неравенство Юнга, получаем

$$
\begin{gathered}
\left(\int_{\Omega(s)}|u|^{1+q} d x\right)^{\xi} \leqslant c d_{3}^{\xi(q+1)} \int_{\Omega(s)}\left(\left|D_{x}^{m} u\right|^{p+1}+|u|^{\lambda+1}\right) d x, \\
\frac{1}{\xi}=\frac{\theta_{1}(1+q)}{(p+1)}+\frac{\left(1-\theta_{1}\right)(q+1)}{\lambda+1}=\frac{n(p-\lambda)+m(p+1)(1+q)}{n(p-\lambda)+m(p+1)(1+\lambda)}>1 .
\end{gathered}
$$

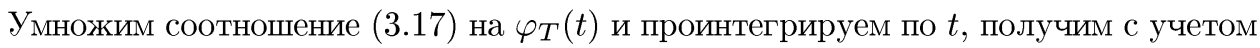
оценки (3.2):

$$
\Psi_{T}(s, \xi) \equiv \int_{0}^{T}\left(\int_{\Omega(s)}|u|^{q+1} d x\right)^{\xi} \varphi_{T}(t) d t \leqslant c J_{T}(s+\delta, \delta) \quad \forall s, \delta \in \mathbb{R}^{1} .
$$

Теперь снова возврашаемся к доказанному в лемме 2 соотношению (3.7). Положим в нем $h=\xi$. Воспользовавшись неравенством

$$
\Phi_{T}(s, h) \leqslant \Psi_{T}(s, h) \quad \forall h>0,
$$

получим

$$
\begin{aligned}
I_{T}(s-\delta) & \leqslant c J_{T}(s, \delta)^{\theta}\left(J_{T}(s+\delta, \delta)^{\frac{p+1}{q+1}-\xi} \Psi_{T}(s, \xi)\right)^{1-\theta} \leqslant c_{1} J_{T}(s+\delta, 2 \delta)^{1+\mu}, \\
\mu & =\left(\frac{p+1}{q+1}-\xi\right)(1-\theta)=\frac{m(p+1)(p-\lambda)}{n(p-\lambda)+m(p+1)(1+q)} .
\end{aligned}
$$

Используя возможность произвольного выбора значений $s$ и $\delta$, теперь просто выводим соотношение

$$
I_{T}(s-\delta) \leqslant c_{2} J_{T}(s, \delta)^{1+\mu} \quad \forall s, \delta \in \mathbb{R}^{1} .
$$

Вспоминая определение функции $J_{T}(s, \delta)$, приходим к оценке

$$
\begin{aligned}
I_{T}(s-\delta) & \leqslant c_{3} \frac{\left(\Delta I_{T}(s)\right)^{1+\mu}}{\delta^{m(p+1)(1+\mu)}}+c_{3} f(s)^{1+\mu} \\
& \leqslant c_{3} \delta^{m(p+1)(1+\mu)} I_{T}(s)^{\mu} \Delta I_{T}(s)+c_{3} f(s)^{1+\mu} \quad \forall T>0 .
\end{aligned}
$$

Полагая в неравенстве $(3.19) \delta=\delta(s) \equiv I_{T}(s)^{\frac{\mu}{m(p+1)(\mu+1)}}$, получаем после стандартных вычислений

$$
I_{T}\left(s-I_{T}(s)^{\frac{\mu}{m(p+1)(\mu+1)}}\right) \leqslant \nu I_{T}(s)+\nu f(s)^{1+\mu}, \quad \nu=\frac{c_{3}}{1+c_{3}}<1 .
$$


Возводя это неравенство в степень $\frac{\mu}{m(p+1)(\mu+1)}$, получим для функции $R_{T}(s) \equiv$ $I_{T}\left(s_{0}+s\right)^{\frac{\mu}{m(p+1)(\mu+1)}}$ соотношение

$$
R_{T}\left(s-R_{T}(s)\right) \leqslant \nu_{1} R_{T}(s)+F(s), \quad \nu_{1}=\nu^{\frac{\mu}{m(p+1)(\mu+1)}}<1,
$$

где $F(s)=\nu_{1} f\left(s_{0}+s\right)^{\frac{\mu}{m(p+1)}}$.

Для анализа соотношения (3.21) снова воспользуемся леммой 4 из дополнения. Аналогично (3.12) устанавливаем априорную оценку

$$
R_{T}(s) \leqslant\left(2 c_{3}\right)^{\frac{\mu}{m(p+1)(\mu+1)}} \nu_{1}^{-1} F(s) \quad \forall s>s^{\prime}-s_{0}, \quad s^{\prime}<\infty .
$$

Для функции $F(s)$ в силу условия $(2.7)$ имеем оценку

$$
F(s) \leqslant \nu_{1} k_{2}^{\frac{\mu}{m(p+1)}} s \quad \forall s>0 .
$$

Теперь в силу соотношения (3.21) и оценок $(3.22),(3.23)$ следует, что $R_{T}(s)=0$ $\forall s \geqslant 0$, если выполняется следуюший аналог условия (5.4):

$$
\nu_{1} k_{2}^{\frac{\mu}{m(p+1)}}<\left(\lambda-\nu_{1}\right)(1-\lambda), \quad \lambda-\nu_{1}=\nu_{1}\left(2 c_{3}\right)^{\frac{\mu}{m(p+1)(\mu+1)}} .
$$

Следовательно, свойство (2.8) будет выполняться, если

$$
k_{2}<k_{2}^{(0)} \equiv\left(2 c_{3}\right)^{-\frac{1}{1+\mu}}\left(1-\nu_{1}-\nu_{1}\left(2 c_{3}\right)^{-\frac{\mu}{m(p+1)(\mu+1)}}\right)^{\frac{m(p+1)}{\mu}} .
$$

Теорема 2 доказана.

ДОКАЗАТЕЛЬСТВО ТЕОРЕМЫ 3 также основано на соотношении (3.7) из леммы 2. Предварительно отметим, что в силу неравенства Гёльдера имеет место оценка

$$
\int_{\Omega(s)}|u|^{p+1} d x \leqslant c\left(\int_{\Omega(s)}|u|^{q+1} d x\right)^{\frac{p-\lambda}{q-\lambda}}\left(\int_{\Omega(s)}|u|^{\lambda+1} d x\right)^{\frac{q-p}{q-\lambda}} \forall s \in \mathbb{R}^{1} .
$$

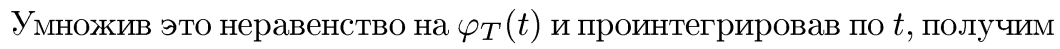

$$
I_{T}(s) \leqslant c_{1}\left(\int_{Q^{(T)}(s)}|u|^{q+1} \varphi_{T} d x d t\right)^{\frac{p-\lambda}{q-\lambda}}\left(\int_{Q^{(T)}(s)}|u|^{\lambda+1} \varphi_{T} d x d t\right)^{\frac{q-p}{q-\lambda}} .
$$

Это неравенство ввиду основной энергетической оценки (3.2) порождает соотношение

$$
I_{T}(s-\delta) \leqslant c_{1} J_{T}(s, \delta)^{\frac{q-p}{q-\lambda}} \Psi_{T}(s-\delta, 1)^{\frac{p-\lambda}{q-\lambda}} \quad \forall s, \delta \in \mathbb{R}^{1} .
$$

$\mathrm{C}$ другой стороны, в силу второго из ограничений (2.9) имеется вложение

$$
W_{p}^{m}(\Omega) \hookrightarrow L_{q}(\Omega)
$$

Поэтому справедливо интерполяционное неравенство (3.16). Следовательно, повторяя рассуждения, аналогичные началу доказательства теоремы 2 , устанавливаем справедливость оценки (3.18) и при соотношениях на параметры уравнения (1.1), 
задаваемых условием (2.9). Из соотношения (3.9) при $h_{2}=1, h_{1}=\xi$, где $\xi<1$ из формулы (3.17), вытекает

$$
\Psi_{T}(s-\delta, 1) \leqslant \Phi_{T}(s, 1) \leqslant c \Phi_{T}(s, \xi) J_{T}(s, \delta)^{1-\xi} .
$$

Так как $\Phi_{T}(s, \xi) \leqslant \Psi_{T}(s, \xi)$, то, продолжая предыдущую оценку при помощи соотношения (3.18), получим

$$
\Psi_{T}(s-\delta, 1) \leqslant c_{1} J_{T}(s+\delta, \delta) J_{T}(s, \delta)^{1-\xi} \quad \forall s, \delta \in \mathbb{R}^{1} .
$$

Подставляя эту оценку в (3.26) и переобозначая аргументы, приходим к следующему:

$$
\begin{aligned}
I_{T}(s-\delta) \leqslant \tilde{c}_{2} J_{T}(s, \delta)^{1+\mu} \quad \forall s, \delta \in \mathbb{R}^{1} ; \\
\mu=(1-\xi) \frac{(p-\lambda)}{(q-\lambda)}=\frac{m(p+1)(p-\lambda)}{n(p-\lambda)+m(p+1)(q+1)} .
\end{aligned}
$$

Вспоминая вид функции $J_{T}(s, \delta)$, выводим из $(3.28)$

$$
I_{T}(s-\delta) \leqslant \tilde{c}_{3} \delta^{-m(p+1)(1+\mu)} I_{T}(s)^{\mu} \Delta I_{T}(s)+\tilde{c}_{3} f(s)^{1+\mu} \quad \forall T>0 .
$$

Продолжая далее анализировать неравенство $\left(3.19^{*}\right)$ точно так же, как неравенство (3.19) в теореме 2, получаем справедливость доказываемого утверждения с

$$
k_{3}^{(0)}=\tilde{\nu}_{1}^{-\frac{m(p+1)}{\mu}}\left(2 \tilde{c}_{3}\right)^{-\frac{1}{1+\mu}}\left(1-\tilde{\nu}_{1}-\left(2 \tilde{c}_{3}\right)^{-\frac{\mu}{m(p+1)(1+\mu)}}\right)^{\frac{m(p+1)}{\mu}},
$$

где $\tilde{\nu}_{1}=\left(\tilde{c}_{3}\left(1+\tilde{c}_{3}\right)^{-1}\right)^{\frac{\mu}{m(p+1)(1+\mu)}}$.

\section{§ 4. Обратное движение фронта}

Введем дополнительные обозначения:

$$
\begin{aligned}
Q_{t_{1}}^{\left(t_{2}\right)}(s) & =\Omega(s) \times\left(t_{1}, t_{2}\right) \quad \forall 0 \leqslant t_{1}<t_{2}<T ; \\
G_{t_{1}}^{\left(t_{2}\right)}(s, \delta) & =Q_{t_{1}}^{\left(t_{2}\right)}(s) \backslash Q_{t_{1}}^{\left(t_{2}\right)}(s-\delta) \quad \forall s, \delta \in \mathbb{R}^{1} .
\end{aligned}
$$

Зафиксируем некоторые числа $0<\tau<T, 0<\omega<\tau$; и пусть $\psi(t) \in C^{1}\left(\mathbb{R}^{1}\right)-$ срезающая функция такая, что $\psi(t)=0$ при $t \leqslant T-\tau, \psi(t)=1$ при $t>T-\tau+\omega$,

$$
|D \psi(t)| \leqslant d_{5} \omega^{-1} \text {. }
$$

ДоКАЗАТЕЛЬСТво ТЕОРЕМЫ 4. Подставим в интегральное тождество (2.1) для изучаемого решения $u(x, t)$ в качестве $\eta$ срезающую функцию $\eta(x)$ из леммы 1 , а в качестве $\varphi$ - срезающую функцию $\psi(t)$ из $(4.1)$, получим исходное равенство

$$
\begin{aligned}
\frac{q}{q+1} & \int_{\Omega(s)}|u(x, T)|^{q+1} \eta d x \\
& +\int_{Q_{T-\tau+\omega}^{(T)}(s)}\left(\sum_{|\alpha|=m} a_{\alpha}\left(x, \ldots, D_{x}^{m} u\right) D^{\alpha} u+b u\right) \eta \psi d x d t \\
= & \frac{q}{q+1} \int_{Q_{T-\tau}^{(T-\tau+\omega)}(s)}|u|^{q+1} \psi_{t}^{\prime}(t) \eta d x d t \\
& -\int_{G_{T-\tau}^{(T)}(s, \delta)} \sum_{|\alpha|=m} a_{\alpha}\left(x, \ldots, D^{m} u\right) \sum_{|\beta| \leqslant m-1} D^{\beta} u D^{\alpha-\beta} \eta \psi d x d t
\end{aligned}
$$


Проводя в этом равенстве преобразования и оценки, аналогичные тем, что проводились в лемме 1 при получении соотношения (3.2), получаем следующее соотношение:

$$
\begin{gathered}
\int_{\Omega(s-\delta)}|u(x, T)|^{q+1} d x+\int_{Q_{T-\tau+\omega}^{(T)}(s-\delta)}\left(\left|D_{x}^{m} u\right|^{p+1}+|u|^{\lambda+1}\right) d x d t \\
\leqslant c_{1}\left(\delta^{-m(p+1)} \int_{G_{T-\tau}^{(T)}(s, \delta)}|u|^{p+1} d x d t\right. \\
\left.+\omega^{-1} \int_{Q_{T-\tau}^{(T-\tau+\omega)}(s)}|u|^{q+1} d x d t\right) \equiv c_{1} Z_{T}(s, \delta, \tau, \omega) .
\end{gathered}
$$

Записывая интерполяционное неравенство (3.1) для функции $u(x, t)$ в области $\Omega(s-\delta)$ при $\alpha=p+1, \beta=p+1, \gamma=q+1, j=0$, возводя его в степень $p+1$ и интегрируя по $t$ в пределах $(T-(\tau-\omega), T)$, получим с учетом оценки $(4.3)$

$$
\begin{aligned}
I_{T}(s-\delta, \tau-\omega) & =\int_{T-(\tau-\omega)}^{T} \int_{\Omega(s-\delta)}|u(x, t)|^{p+1} d x d t \\
& \leqslant c Z_{T}(s, \delta, \tau, \omega)^{\theta}\left(\Psi_{T-(\tau-\omega)}^{(T)}\left(s-\delta, \frac{p+1}{q+1}\right)\right)^{1-\theta},
\end{aligned}
$$

где $0<\theta<1$ из соотношения (3.7),

$$
\Psi_{w}^{(v)}(s, h) \equiv \int_{w}^{v}\left(\int_{\Omega(s)}|u(x, t)|^{q+1} d x\right)^{h} d t
$$

Оцениваем теперь отдельно второй сомножитель в правой части неравенства (4.4). Во-первых, интегрируя неравенство (3.17) в пределах $(T-(\tau-\omega), T)$ и используя оценку (4.3), получаем

$$
\Psi_{T-(\tau-\omega)}^{(T)}(s-\delta, \xi) \leqslant c Z_{T}(s, \delta, \tau, \omega)
$$

Теперь по аналогии с леммой 2 установим соотношение между функциями $\Psi_{T-(\tau-\omega)}^{(T)}(s, h)$ при различных $h>0$. Введем вспомогательную функцию

$$
\chi^{(h)}(t)=\int_{0}^{t}\left(\int_{\Omega(s)}|u(x, \theta)|^{q+1} \eta\left(x_{n}\right) \psi(\theta) d x\right)^{h} d \theta
$$

где $\eta\left(x_{n}\right), \psi(t)$ - срезающие функции из тождества (4.2). В силу теоремы Фубини очевидно следующее равенство:

$$
\begin{aligned}
\Phi_{T-\tau}^{(T)}(s, h+1) & \equiv \chi^{(h+1)}(T) \\
& =\int_{Q^{(T)}(s)}|u(x, t)|^{q+1} \eta\left(x_{n}\right) \psi(t) \frac{d}{d t}\left(\chi^{(h)}(t)\right) d x d t .
\end{aligned}
$$


Поэтому, подставляя в интегральное тождество (2.1) в качестве $\eta$ срезающую функцию $\eta\left(x_{n}\right)$ из леммы 1 , а в качестве $\varphi(t)$ функцию $\psi(t) \chi^{(h)}(t)$, получим с учетом (4.6)

$$
\begin{gathered}
\Phi_{T-\tau}^{(T)}(s, h+1)=\Phi_{T-\tau}^{(T)}(s, h) \int_{\Omega(s)}|u(x, T)|^{q+1} \eta d x \\
+\frac{q}{q+1} \int_{Q_{T-\tau}^{(T)}(s)}\left(\sum_{|\alpha|=m} a_{\alpha}\left(u, \ldots, D^{m} u\right) D^{\alpha}(u \eta)+b(x, t, u) u \eta\right) \psi(t) \chi^{(h)}(t) d x d t \\
-\int_{Q_{T-\tau}^{(T-\tau+\omega)}(s)}|u|^{q+1} \eta \psi_{t}^{\prime} \chi^{(h)}(t) d x d t .
\end{gathered}
$$

Отсюда, используя то, что $\psi_{t}^{\prime} \geqslant 0$, условия (1.5), (1.6), оценку (3.3), интерполяционное неравенство (3.1) при $\alpha=\beta=\gamma=p+1, j=0,1, \ldots, m-1$, а также установленную выше оценку (4.3) с $s+\delta$ вместо $s, \tau+\omega$ вместо $\tau$, получаем соотношение

$$
\Phi_{T-\tau}^{(T)}(s, h+1) \leqslant c \Phi_{T-\tau}^{(T)}(s, h) Z_{T}(s+\delta, \delta, \tau+\omega, \omega) \quad \forall h>0 .
$$

Отсюда с помощью неравенства Гёльдера получаем

$$
\Phi_{T-\tau}^{(T)}\left(s, h_{2}\right) \leqslant c \Phi_{T-\tau}^{(T)}\left(s, h_{1}\right) Z_{T}(s+\delta, \delta, \tau+\omega, \omega)^{h_{2}-h_{1}} \quad \forall h_{2}>h_{1}>0 .
$$

В частности, при $h_{2}=\frac{p+1}{q+1}, h_{1}=\xi$ имеем

$$
\begin{aligned}
\Psi_{T-\tau+\omega}^{(T)}\left(s-\delta, \frac{p+1}{q+1}\right) & \leqslant \Phi_{T-\tau}^{(T)}\left(s, \frac{p+1}{q+1}\right) \\
& \leqslant c \Phi_{T-\tau}^{(T)}(s, \xi) Z_{T}(s+\delta, \delta, \tau+\omega, \omega)^{\frac{p+1}{q+1}-\xi}
\end{aligned}
$$

Наконец, первый сомножитель справа оценим, используя неравенство (4.5) с соответствуюшим образом замененными аргументами:

$$
\Phi_{T-\tau}^{(T)}(s, \xi) \leqslant \Psi_{T-\tau}^{(T)}(s, \xi) \leqslant c Z_{T}(s+\delta, \delta, \tau+\omega, \omega) .
$$

Подставляя эту оценку в (4.8), получаем

$$
\Psi_{T-\tau+\omega}^{(T)}\left(s-\delta, \frac{p+1}{q+1}\right) \leqslant c Z_{T}(s+\delta, \delta, \tau+\omega, \omega)^{1+\frac{p+1}{q+1}-\xi} .
$$

Используя это соотношение в неравенстве (4.4), приходим к

$$
\begin{gathered}
I_{T}(s-\delta, \tau-\omega)=c Z_{T}(s, \delta, \tau, \omega)^{\theta} Z_{T}(s+\delta, \delta, \tau+\omega, \omega)^{1-\theta} \\
\times Z_{T}(s+\delta, \delta, \tau+\omega, \omega)^{\mu} \\
\mu=\left(\frac{p+1}{q+1}-\xi\right)(1-\theta)=\frac{m(p+1)(p-\lambda)}{m(p+1)(q+1)+n(p-\lambda)}
\end{gathered}
$$

Делая в последнем неравенстве стандартную замену аргументов: $s+\delta \rightarrow s_{1}$, $2 \delta \rightarrow \delta_{1}, \tau+\omega \rightarrow \tau_{1}, 2 \omega \rightarrow \omega_{1}$, и затем опуская индекс, получаем основное соотношение

$$
I_{T}(s-\delta, \tau-\omega) \leqslant c Z_{T}(s, \delta, \tau, \omega)^{1+\mu} \quad \forall s, \delta \in \mathbb{R}^{1}, \quad 0<\omega<\tau \leqslant T .
$$


Введем еше одно обозначение

$$
J_{T}(s, \tau) \equiv \int_{T-\tau}^{T} \int_{\Omega(s)}|u(x, t)|^{q+1} d x d t=\Psi_{T-\tau}^{(T)}(s, 1) .
$$

Для функции $J_{T}(s, \tau)$ установим некоторое соотношение, аналогичное неравенству (4.9) для $I_{T}(s, \tau)$. Полагая в соотношении $(4.7) h_{2}=1, h_{1}=\xi$ и используя также оценку (4.5), получаем

$$
\begin{aligned}
J_{T}(s-\delta, \tau-\omega) & \leqslant \Phi_{T-\tau}^{(T)}(s, 1) \leqslant c \Phi_{T-\tau}^{(T)}(s, \xi) Z_{T}(s+\delta, \delta, \tau+\omega, \omega)^{1-\xi} \\
& \leqslant c Z_{T}(s+\delta, \delta, \tau+\omega, \omega)^{2-\xi} .
\end{aligned}
$$

Это неравенство после стандартной замены аргументов дает

$$
\begin{aligned}
& J_{T}(s-\delta, \tau-\omega) \leqslant c Z_{T}(s, \delta, \tau, \omega)^{1+\nu}, \\
& \nu=1-\xi=\frac{m(p+1)(q-\lambda)}{m(p+1)(q+1)+n(p-\lambda)} .
\end{aligned}
$$

Определим также два разностных оператора:

$$
\begin{aligned}
\Delta_{s} I_{T}(s, \tau) & =I_{T}(s, \tau)-I_{T}(s-\delta, \tau), \\
\Delta_{\tau} J_{T}(s, \tau) & =J_{T}(s, \tau)-J_{T}(s, \tau-\omega) .
\end{aligned}
$$

Тогда функция $Z_{T}(s, \delta, \tau, \omega)$ может быть записана в виде

$$
Z_{T}(s, \delta, \tau, \omega)=\delta^{-m(p+1)} \Delta_{s} I_{T}(s, \tau)+\omega^{-1} \Delta_{\tau} J_{T}(s, \tau) .
$$

Возведем теперь неравенства (4.9) и (4.10) соответственно в степень $1+\nu$ и $1+\mu$ и сложим. В результате имеем для функции

$$
H_{T}(s, \tau) \equiv I_{T}(s, \tau)^{1+\nu}+J_{T}(s, \tau)^{1+\mu} \equiv A_{T}(s, \tau)+B_{T}(s, \tau)
$$

соотношение

$$
H_{T}(s-\delta, \tau-\omega) \leqslant c\left(\frac{\Delta_{s} I_{T}(s, \tau)}{\delta^{m(p+1)}}+\frac{\Delta_{\tau} J_{T}(s, \tau)}{\omega}\right)^{(1+\nu)(1+\mu)} .
$$

Используя просто проверяемые неравенства

$$
\begin{array}{ll}
\left(\Delta_{s} I_{T}(s, \tau)\right)^{1+\alpha} \leqslant I_{T}(s, \tau)^{1+\alpha}-I_{T}(s-\delta, \tau)^{1+\alpha} & \forall \alpha \geqslant 0, \\
\left(\Delta_{\tau} J_{T}(s, \tau)\right)^{1+\beta} \leqslant J_{T}(s, \tau)^{1+\beta}-J_{T}(s, \tau-\omega)^{1+\beta} & \forall \beta \geqslant 0,
\end{array}
$$

выводим из соотношения (4.12)

$$
\begin{aligned}
H_{T}(s-\delta, \tau-\omega) & \leqslant c_{1} \frac{\left(\Delta_{s} A_{T}(s, \tau)\right)^{1+\mu}}{\delta^{m(p+1)(1+\nu)(1+\mu)}}+c_{1} \frac{\left(\Delta_{\tau} B_{T}(s, \tau)\right)^{1+\nu}}{\omega^{(1+\nu)(1+\mu)}} \\
& \leqslant c_{1} \frac{A_{T}(s, \tau)^{\mu} \Delta_{s} A_{T}(s, \tau)}{\delta^{m(p+1)(1+\nu)(1+\mu)}}+c_{1} \frac{B_{T}(s, \tau)^{\nu} \Delta_{\tau} B_{T}(s, \tau)}{\omega^{(1+\nu)(1+\mu)}} .
\end{aligned}
$$

Определим теперь величины приращений $\delta, \omega$ :

$$
\delta=\delta(s, \tau) \equiv A_{T}(s, \tau)^{\frac{\mu}{m(p+1)(1+\nu)(1+\mu)}} ; \quad \omega=\omega(s, \tau) \equiv B_{T}(s, \tau)^{\frac{\nu}{(1+\nu)(1+\mu)}} .
$$


Теперь, учитьвая монотонность функций $A_{T}(s, \tau), B_{T}(s, \tau)$ по обоим аргументам, легко выводим из последнего неравенства

$$
H_{T}(s-\delta(s, \tau), \tau-\omega(s, \tau)) \leqslant \theta H_{T}(s, \tau) ; \quad \theta=\frac{c_{1}}{1+c_{1}}<1 .
$$

Наконец, используя монотонность функции $H_{T}(s, \tau)$ по обоим аргументам, получаем окончательное соотношение

$$
H_{T}\left(s-H_{T}(s, \tau)^{\frac{\mu}{m(p+1)(1+\nu)(1+\mu)}}, \tau-H_{T}(s, \tau)^{\frac{\nu}{(1+\nu)(1+\mu)}}\right) \leqslant \theta H_{T}(s, \tau) .
$$

В силу леммы 3 из дополнения из неравенства (4.13) вытекает, что для любого $s^{\prime}<\infty$

$$
\begin{aligned}
& H_{T}(s, \tau) \equiv 0 \\
& \forall(s, \tau) \in\left\{s<s^{\prime}-\left(1-\theta^{\frac{\mu}{m(p+1)(\mu+1)(\nu+1)}}\right)^{-1} H_{T}\left(s^{\prime}, \tau\right)^{\frac{\mu}{m(p+1)(\mu+1)(\nu+1)}},\right. \\
& \left.\tau<T-\left(1-\theta^{\frac{v}{(\mu+1)(\nu+1)}}\right)^{-1} H_{T}\left(s^{\prime}, T\right)^{\frac{v}{(\mu+1)(\nu+1)}}\right\} .
\end{aligned}
$$

Вспоминая теперь определение функции $H_{T}(s, \tau)$, выводим из соотношения (4.14) после простых арифметических выкладок:

$$
\begin{aligned}
& u(x, t) \equiv 0 \\
& \forall(x, t) \in\left\{x_{n}<s^{\prime}-\tilde{c}_{1}\left(1-\theta^{\frac{\mu}{m(p+1)(\mu+1)(\nu+1)}}\right)^{-1}\right. \\
& \quad \times\left(I_{T}^{(0)}\left(s^{\prime}\right)^{\frac{\mu}{m(p+1)(\mu+1)}}+J_{T}^{(0)}\left(s^{\prime}\right)^{\frac{\mu}{m(p+1)(\nu+1)}}\right), \\
& \left.t>\tilde{c}_{2}\left(1-\theta^{\frac{\nu}{(\mu+1)(\nu+1)}}\right)^{-1}\left(I_{T}^{(0)}\left(s^{\prime}\right)^{\frac{\nu}{(\mu+1)}}+J_{T}^{(0)}\left(s^{\prime}\right)^{\frac{\nu}{(\nu+1)}}\right)\right\},
\end{aligned}
$$

где

$$
I_{T}^{(0)}(s)=\int_{Q^{(T)}(s)}|u(x, t)|^{p+1} d x d t, \quad J_{T}^{(0)}(s)=\int_{Q^{(T)}(s)}|u(x, t)|^{q+1} d x d t .
$$

Теперь наша задача - получить оценку сверху энергетических функций $I_{T}^{(0)}(s)$, $J_{T}^{(0)}(s)$ через функцию $f(s)$, характеризующую начальное значение $u_{0}(x)$. Заметим, что из соотношения (3.19), установленного в теореме 2 , следует неравенство

$$
I_{T}^{(0)}(s-\delta) \leqslant e I_{T}(s-\delta) \leqslant e c_{3} \frac{\left(\Delta I_{T}^{(0)}(s)\right)^{1+\mu}}{\delta^{m(p+1)(\mu+1)}}+e c_{3} f(s)^{1+\mu} .
$$

Следуя вычислениям, проведенным в (3.19)-(3.21), выведем из соотношения (4.16) неравенство

$$
R_{T}\left(s-R_{T}(s)\right) \leqslant \theta_{1} R_{T}(s)+F(s) \quad \forall s<\infty,
$$

где

$$
\begin{aligned}
R_{T}(s) & =I_{T}^{(0)}\left(s_{0}+s\right)^{\frac{\mu}{m(p+1)(\mu+1)}}, \\
F(s) & =\theta_{1} f\left(s_{0}+s\right)^{\frac{\mu}{m(p+1)}}, \\
\theta_{1} & =\left(\frac{e c_{3}}{1+e c_{3}}\right)^{\frac{\mu}{m(p+1)(\mu+1)}}<1 .
\end{aligned}
$$


Анализ соотношения (4.17) проведем на основе леммы 5 из дополнения. В силу условия (2.7) имеем

$$
F(s) \leqslant \theta_{1} k_{2}^{\frac{\mu}{m(p+1)}} s \equiv F_{0}(s), \quad f_{0}(s)=k_{2}\left(s-s_{0}\right)^{\alpha_{2}} .
$$

Аналогично (3.12) устанавливаем априорную оценку

$$
\begin{aligned}
R_{T}(s) & \leqslant K_{1}^{(0)} F(s) \leqslant K_{1}^{(0)} F_{0}(s) \quad \forall s>\left(s^{\prime}-s_{0}\right), \\
s^{\prime} & <\infty, \quad K_{1}^{(0)}=\theta_{1}^{-1}\left(2 e c_{3}\right)^{\frac{\mu}{m(p+1)(\mu+1)}} .
\end{aligned}
$$

Зафиксируем теперь постоянную $K_{1}<\infty$ :

$$
K_{1}>\max \left(K_{1}^{(0)},\left(1-\theta_{1}\right)^{-1}\right),
$$

где $0<\theta_{1}<1$ из $(4.17)$.

Пусть также выполняется аналог условия (5.4):

$$
\theta_{1} k_{2}^{\frac{\mu}{m(p+1)}}<K_{1}^{-1}\left(1-\theta_{1}-K_{1}^{-1}\right) .
$$

При этом, как несложно проверить (см. замечание 5 к лемме 5 ), функция $F_{0}(s)$ из (4.18) удовлетворяет условию квалифицированной монотонности (5.8), т.е.

$$
F_{0}\left(s-K_{1} F_{0}(s)\right) \geqslant\left(\theta_{1}+K_{1}^{-1}\right) F_{0}(s) \quad \forall s>0 .
$$

Поэтому в силу леммы 5 справедлива оценка

$$
R_{T}(s) \leqslant K_{1} F_{0}(s) \quad \forall s>0
$$

или

$$
I_{T}^{(0)}(s) \leqslant\left(\theta_{1} K_{1}\right)^{1+\mu} f_{0}(s)^{1+\mu} \quad \forall s \geqslant s_{0} .
$$

Теперь оценим функцию $J_{T}^{(0)}(s)$. Установленное в теореме 2 соотношение (3.27) дает

$$
J_{T}^{(0)}(s-\delta) \leqslant e \Psi_{T}(s-\delta, 1) \leqslant e c_{1}\left(J_{T}(s+\delta, 2 \delta)\right)^{2-\xi} .
$$

Это неравенство в силу того, что $1-\xi=\nu$, после стандартных вычислений приводит к неравенству

$$
J_{T}^{(0)}(s-\delta) \leqslant e c_{1}\left(\frac{\Delta I_{T}^{(0)}(s)}{\delta^{m(p+1)}}+f(s)\right)^{1+\nu} \quad \forall s, \delta \in \mathbb{R}^{1} .
$$

Положим в этом соотношении

$$
\delta=K_{1} F_{0}\left(s-s_{0}\right)
$$

7

Серия математическая, №3 
а для оценки сверху $\Delta I_{T}^{(0)}(s)$ воспользуемся полученной оценкой (4.23). В результате простых вычислений, использующих свойство (4.22), получим

$$
\begin{aligned}
J_{T}^{(0)}(s & \left.-K_{1} F_{0}\left(s-s_{0}\right)\right) \leqslant e c_{1}\left(\frac{\left(\theta_{1} K_{1}\right)^{1+\mu} f_{0}(s)^{1+\mu}}{K_{1}^{m(p+1)} F_{0}\left(s-s_{0}\right)^{m(p+1)}}+f_{0}(s)\right)^{1+\nu} \\
& =e c_{1} f_{0}(s)^{1+\nu}\left(1+\left(\theta_{1} K_{1}\right)^{1+\mu-m(p+1)}\right)^{1+\nu} \\
& =F_{0}\left(s-s_{0}\right)^{\frac{m(p+1)(1+\nu)}{\mu}} \frac{e c_{1}}{\theta_{1}^{\frac{m(p+1)(1+\nu)}{\mu}}}\left(1+\left(\theta_{1} K_{1}\right)^{1+\mu-m(p+1)}\right)^{1+\nu} \\
& \leqslant \frac{e c_{1}\left(1+\left(\theta_{1} K_{1}\right)^{1+\mu-m(p+1)}\right)^{1+\nu} F_{0}\left(s-s_{0}-K_{1} F_{0}\left(s-s_{0}\right)\right)^{\frac{m(p+1)(1+\nu)}{\mu}}}{\theta_{1}^{\frac{m(p+1)(1+\nu)}{\mu}}\left(\theta_{1}+K_{1}^{-1}\right)^{\frac{m(p+1)(1+\nu)}{\mu}}}
\end{aligned}
$$

Вспоминая определение функции $F_{0}(s)$, получаем оценку

$$
\begin{aligned}
J_{T}^{(0)}\left(s-K_{1} F_{0}\left(s-s_{0}\right)\right) & \leqslant K_{2} f_{0}\left(s-K_{1} F_{0}\left(s-s_{0}\right)\right)^{1+\nu} \\
K_{2} & =\frac{e c_{1}\left(1+\left(\theta_{1} K_{1}\right)^{1+\mu-m(p+1)}\right)^{1+\nu}}{\left(\theta_{1}+K_{1}^{-1}\right)^{\frac{m(p+1)(1+\nu)}{\mu}}} .
\end{aligned}
$$

Так как $s-K_{1} F_{0}\left(s-s_{0}\right)=s\left(1-K_{1} \theta_{1} k_{2}^{\frac{\mu}{m(p+1)}}\right)$, причем в силу $(4.21)$

$$
1-K_{1} \theta_{1} k_{2}^{\frac{\mu}{m(p+1)}}>\theta_{1}+K_{1}^{-1}>0
$$

то из оценки (4.25) окончательно выводим

$$
J_{T}^{(0)}(s) \leqslant K_{2} f_{0}(s)^{1+\nu} \quad \forall s \geqslant s_{0} .
$$

Объединяя оценки (4.23) и (4.26), получаем

$$
I_{T}^{(0)}(s)^{\frac{\mu}{m(p+1)(1+\mu)}}+J_{T}^{(0)}(s)^{\frac{\mu}{m(p+1)(1+\nu)}} \leqslant K_{3} f_{0}(s)^{\frac{\mu}{m(p+1)}}
$$

где

$$
K_{3}=\left(\theta_{1} K_{1}\right)^{\frac{\mu}{m(p+1)}}+K_{2}^{\frac{\mu}{m(p+1)(1+\nu)}}
$$

Аналогично получаем

$$
I_{T}^{(0)}(s)^{\frac{\nu}{\mu+1}}+J_{T}^{(0)}(s)^{\frac{\nu}{\nu+1}} \leqslant K_{4} f_{0}(s)^{\nu}, \quad K_{4}=\left(\theta_{1} K_{1}\right)^{\nu}+K_{2}^{\frac{\nu}{\nu+1}}
$$

Подставляя оценки (4.27), (4.28) в (4.15), получаем

$$
\begin{aligned}
u(x, t) & \equiv 0 \quad \forall(x, t) \in\left\{x_{n}<s^{\prime}-\frac{c_{1} K_{3} f_{0}\left(s^{\prime}\right)^{\frac{\mu}{m(p+1)}}}{1-\theta^{\frac{\mu}{m(p+1)(1+\mu)(1+\nu)}}}=s_{0}+\left(s^{\prime}-s_{0}\right)\right. \\
& \left.\times\left(1-\frac{c_{1} K_{3} k_{2}^{\frac{\mu}{m(p+1)}}}{1-\theta^{\frac{\mu}{m(p+1)(1+\mu)(1+\nu)}}}\right), t>\frac{c_{2} K_{4} k_{2}^{\nu}\left(s^{\prime}-s_{0}\right)^{\frac{m(p+1)(q-\lambda)}{p-\lambda}}}{1-\theta^{\frac{\nu}{(1+\mu)(1+\nu)}}}\right\} .
\end{aligned}
$$


Определим теперь $k_{2}^{(1)}>0$ формулой

$$
k_{2}^{(1)}=\left(\min \left\{\theta_{1}^{-1} K_{1}^{-1}\left(1-\theta_{1} K_{1}^{-1}\right), \quad c_{1}^{-1} K_{3}^{-1}\left(1-\theta^{\frac{\mu}{m(p+1)(1+\mu)(1+\nu)}}\right)\right\}\right)^{\frac{m(p+1)}{\mu}} .
$$

Очевидно, что для любого $k_{2}<k_{2}^{(1)}$ выполняется условие (4.2), а также

$$
\omega\left(k_{2}\right) \equiv 1-\frac{c_{1} K_{3} k_{2}^{\frac{\mu}{m(p+1)}}}{1-\theta^{\frac{\mu}{m(p+1)(1+\mu)(1+\nu)}}}>0 \quad \forall k_{2}: 0<k_{2}<k_{2}^{(1)} .
$$

Поэтому при выполнении условия (2.7) с $k_{2}<k_{2}^{(1)}$ из соотношения (4.29) следует, что в качестве искомой функции $l(t)$, описывающей движение носителя решения $u(x, t)$, может быть взята

$$
l(t) \equiv s_{0}+\omega\left(k_{2}\right) K_{5}^{-\frac{p-\lambda}{m(p+1)(q-\lambda)}} t^{\frac{p-\lambda}{m(p+1)(q-\lambda)}}, \quad K_{5} \equiv \frac{c_{2} K_{4} k_{2}^{\nu}}{1-\theta^{\frac{\nu}{(1+\mu)(1+\nu)}}} .
$$

Первое утверждение теоремы 4 доказано. Для проверки второго утверждения следует уточнить оценки функций $I_{T}^{(0)}(s), J_{T}^{(0)}(s)$, удовлетворяющих соответственно соотношениям (4.16) и (4.24). Вытекаюшее из (4.16) соотношение (4.17) дает

$$
R_{T}\left(s-R_{T}(s)\right) \leqslant \theta_{1} R_{T}(s)+F_{1}(s) \quad \forall s<\infty, \quad F_{1}(s)=\theta_{1} f_{1}\left(s_{0}+s\right)^{\frac{\mu}{m(p+1)}} .
$$

Отсюда в силу леммы 5 из дополнения следует

$$
R_{T}(s) \leqslant K_{1} F_{1}(s) \quad \forall s>0,
$$

если $F_{1}(s)$ удовлетворяет условию квалифицированной монотонности:

$$
F_{1}\left(s-K_{1} F_{1}(s)\right) \geqslant\left(\theta_{1}+K_{1}^{-1}\right) F_{1}(s),
$$

которое эквивалентно

$$
f_{1}\left(s_{0}-s-K_{1} \theta_{1} f_{1}\left(s_{0}+s\right)^{\frac{\mu}{m(p+1)}}\right) \geqslant\left(\theta_{1}+K_{1}^{-1}\right)^{\frac{m(p+1)}{\mu}} f_{1}\left(s_{0}+s\right) .
$$

Полагая в условии (2.13) $D=K_{1} \theta_{1}, d=\theta_{1}+K_{1}^{-1}$, получаем выполнение условия (4.32). Следовательно, справедлива оценка (4.31), а значит, и эквивалентная ей оценка

$$
I_{T}^{(0)}(s) \leqslant\left(K_{1} \theta_{1}\right)^{1+\mu} f_{1}(s)^{1+\mu} \quad \forall s>s_{0} .
$$

Подставляя эту оценку в соотношение (4.24) и действуя так же, как при выводе оценки (4.26), получаем

$$
J_{T}^{(0)}(s) \leqslant K_{2} f_{1}(s)^{1+\nu} \quad \forall s>s_{0} .
$$

Используя оценки (4.33), (4.34) в формуле (4.15), получаем

$$
u(x, t) \equiv 0 \quad \forall(x, t) \in\left\{x_{n} \leqslant s_{0}+\omega\left(k_{2}\right)\left(s^{\prime}-s_{0}\right), \quad t \geqslant \frac{K_{5}}{k_{2}^{\nu}} f_{1}\left(s^{\prime}\right)^{\nu}\right\} .
$$

Отсюда вытекает оценка для $l(t)$ :

$$
l(t) \geqslant s_{0}+\omega\left(k_{2}\right)\left[f_{1}^{-1}\left(k_{2} K_{5}^{-\frac{1}{\nu}} t^{\frac{1}{\nu}}\right)-s_{0}\right] .
$$

Теорема 4 доказана полностью. 
ДОКАЗАТЕЛЬСТВО ТЕОРЕМЫ 5 проводится комбинацией рассуждений, проводившихся при выводе теорем 3 и 4 . Интегрируя неравенство (3.25) в пределах $(T-(\tau-\omega), T)$ и используя установленное соотношение $(4.3)$, получаем

$$
I_{T}(s-\delta, \tau-\omega) \leqslant c Z_{T}(s, \delta, \tau, \omega)^{\frac{q-p}{q-\lambda}} \Psi_{T-(\tau-\omega)}^{(T)}(s-\delta, 1)^{\frac{p-\lambda}{q-\lambda}},
$$

где, как и ранее,

$$
I_{T}(s, v) \equiv \int_{T-v}^{T} d t\left(\int_{\Omega(s)}|u(x, t)|^{p+1} d x\right)
$$

$Z_{T}(s, \delta, \tau, \omega), \Psi_{T-v}^{T}(s, h)-$ из (4.3), (4.4).

Так как в силу условий (2.9) справедливо интерполяционное неравенство (3.16), то, интегрируя вытекающее из него соотношение (3.17) в пределах $(T-(\tau-\omega), T)$, получим после дополнительного применения основного соотношения (4.3)

$$
\Psi_{T-\tau}^{(T)}(s, \xi) \leqslant c Z_{T}(s+\delta, \delta, \tau+\omega, \omega),
$$

где $\xi<1$ из соотношения (3.17). Теперь стандартным образом устанавливаем справедливость при соотношениях (2.9) на параметры $\lambda, p, q$ неравенства (4.7), характеризующего соотношение между функциями

$$
\Phi_{v}^{(T)}(s, h) \equiv \int_{T-v}^{T} d t\left(\int_{\Omega(s)}|u(x, t)|^{q+1} \eta\left(x_{n}\right) \psi(t) d x\right)^{h}
$$

при различных значениях $h>0$. Из этого соотношения вытекает

$$
\begin{aligned}
& \Psi_{T-(\tau-\omega)}^{T}(s-\delta, 1) \leqslant \Phi_{T-\tau}^{(T)}(s, 1) \\
& \quad \leqslant c \Phi_{T-\tau}^{(T)}(s, \xi) Z_{T}(s+\delta, \delta, \tau+\omega, \omega)^{1-\xi} \quad \forall s, \delta \in \mathbb{R}^{1} .
\end{aligned}
$$

Так как $\Phi_{T-\tau}^{(T)}(s, \xi) \leqslant \Psi_{T-\tau}^{(T)}(s, \xi)$, то, объединяя оценки (4.37), (4.36), получаем

$$
\Psi_{T-(\tau-\omega)}^{T}(s-\delta, 1) \leqslant Z_{T}(s+\delta, \delta, \tau+\omega, \omega)^{2-\xi} .
$$

Подставляя эту оценку в соотношение (4.35), получаем после стандартной замены аргументов

$$
\begin{gathered}
I_{T}(s-\delta, \tau-\omega) \leqslant c Z_{T}(s, \delta, \tau, \omega)^{1+\mu}, \\
\mu=(1-\xi) \frac{(p-\lambda)}{(q-\lambda)}=\frac{m(p+1)(p-\lambda)}{n(p-\lambda)+m(p+1)(q+1)} .
\end{gathered}
$$

Само неравенство (4.38) после замены аргументов дает

$$
J_{T}(s-\delta, \tau-\omega) \equiv \Psi_{T-(\tau-\omega)}^{T}(s-\delta, 1) \leqslant c_{1} Z_{T}(s, \delta, \tau, \omega)^{1+\nu},
$$

где

$$
\nu=1-\xi=\frac{m(p+1)(q-\lambda)}{n(p-\lambda)+m(p+1)(q+1)} .
$$

Дальнейшее доказательство практически полностью повторяет соответствующую часть теоремы 4. Из соотношений (4.39), (4.40) для функции $H_{T}(s, \tau)=$ $I_{T}(s, \tau)^{1+\nu}+J_{T}(s, \tau)^{1+\mu}$ устанавливаем основное неравенство (4.13) с некоторым 
новым $\theta: 0<\theta<1$. Следовательно, имеет место и оценка (4.15) носителя решения $u(x, t)$. После этого остается оценить функции $I_{T}^{(0)}(s), J_{T}^{(0)}(s)$. Для $I_{T}^{(0)}(s)$ в силу соотношения (3.28) из теоремы 3 имеем соотношение

$$
I_{T}^{(0)}(s-\delta) \leqslant e \tilde{c}_{2}\left(\frac{\Delta I_{T}^{(0)}(s)}{\delta^{m(p+1)}}\right)^{1+\mu}+e \tilde{c}_{2} f(s)^{1+\mu} .
$$

Из этого соотношения так же, как и в теореме 4 , получаем, используя лемму 4 из дополнения, явную оценку

$$
I_{T}^{(0)}(s) \leqslant\left(\widetilde{K}_{1} \tilde{\theta}_{1}\right)^{1+\mu} f_{0}(s)^{1+\mu} \quad \forall s>s_{0}, \quad f_{0}(s)=k_{3}\left(s-s_{0}\right)^{\alpha_{2}} .
$$

Для оценки $J_{T}^{(0)}(s)$ используем вытекающее из доказанного в теореме 3 соотношения (3.27) неравенство

$$
J_{T}^{(0)}(s-\delta) \leqslant c_{1} e\left(\frac{\Delta I_{T}^{(0)}(s)}{\delta^{m(p+1)}}+f_{0}(s)\right)^{1+\nu} .
$$

Из этого соотношения и оценки (4.42), действуя точно так же, как при выводе оценки (4.26), получаем

$$
J_{T}^{(0)}(s) \leqslant \widetilde{K}_{2} f_{0}(s)^{1+\nu} \quad \forall s>s_{0}, \widetilde{K}_{2}<\infty .
$$

Теперь из соотношения (4.15) и оценок $(4.42),(4.43)$ следует свойство (2.11) с необходимой функцией $l(t)>0$ из (2.14). Теорема 5 доказана.

\section{$\S 5$. Дополнение}

ЛЕмма 3 [21]. Пусть некоторая неотрицательная непрерьвная функция $f\left(t_{1}, \ldots, t_{m}\right), m \geqslant 1$, не убшвает по каждому из своих аргументов и удовлетворяет следующему соотношению:

$$
\begin{aligned}
& f\left(t_{1}-c_{1} f\left(t_{1}, \ldots, t_{m}\right)^{\nu_{1}}, t_{2}-c_{2} f\left(t_{1}, \ldots, t_{m}\right)^{\nu_{2}}, \ldots, t_{m}-c_{m} f\left(t_{1}, \ldots, t_{m}\right)^{\nu_{m}}\right) \\
& \quad \leqslant \varepsilon f\left(t_{1}, \ldots, t_{m}\right) \quad \forall t_{i}<t_{i}^{(0)} ; \quad c_{i}>0, \quad \nu_{i}>0, \quad i=1,2, \ldots, m ; \quad 0<\varepsilon<1 .
\end{aligned}
$$

Тогда

$$
\begin{aligned}
& f\left(t_{1}, \ldots, t_{m}\right) \equiv 0 \\
& \forall\left(t_{1}, \ldots, t_{m}\right) \in\left\{t_{i} \leqslant t_{i}^{(0)}-\frac{c_{i}}{1-\varepsilon^{\nu_{i}}} f\left(t_{1}^{(0)}, \ldots, t_{m}^{(0)}\right)^{\nu_{i}}, \quad i=1,2, \ldots, m\right\} .
\end{aligned}
$$

Лемма 4. Пусть некоторая непрерывная неотрицательная неубывающая функщия $f(s)$ удовлетворяет следующему функииональному соотношению:

$$
f(s-f(s)) \leqslant \varepsilon f(s)+g(s) \quad \forall s \in \mathbb{R}^{1}, \quad 0<\varepsilon<1,
$$

где известная неотрицательная непрерывная неубывающая функщия $g(s)$ удовлетворяет оценке

$$
g(s) \leqslant a s \quad \forall s>0, \quad 0<a<\infty .
$$

Пусть также для некоторого $s_{0}^{\prime}<\infty$ выполняется следующее соотношение:

$$
f(s) \leqslant k g(s) \quad \forall s>s_{0}^{\prime}>0, k>(1-\varepsilon)^{-1} .
$$

Тогда если для параметра а из соотношения (5.2) выполняется ограничение

$$
a<k^{-1}\left(1-\varepsilon-k^{-1}\right),
$$

$m o f(s) \equiv 0 \quad \forall s \leqslant 0$. 
ДокАЗАТЕЛЬСтво. Предположим противное, т.е. $f(0)>0$. Тогда, конечно, существует $s^{\prime}>0$ такое, что

$$
f(s)>(\lambda-\varepsilon)^{-1} g(s) \equiv k g(s), \quad \varepsilon<\lambda<1, \quad \forall s: 0<s<s^{\prime} .
$$

В силу (5.3) можно считать, что

$$
f\left(s^{\prime}\right)=(\lambda-\varepsilon)^{-1} g\left(s^{\prime}\right), \quad s^{\prime} \leqslant s_{0}^{\prime} .
$$

Из соотношения (5.1) в силу предположения (5.5) вытекает

$$
f(s-f(s)) \leqslant \varepsilon f(s)+(\lambda-\varepsilon) f(s)=\lambda f(s) \quad \forall s: 0<s<s^{\prime} .
$$

В силу (5.6), (5.4), (5.2) имеем следуюшее соотношение:

$$
\frac{f\left(s^{\prime}\right)}{1-\lambda}=\frac{g\left(s^{\prime}\right)}{(1-\lambda)(\lambda-\varepsilon)}<\frac{g\left(s^{\prime}\right)}{a} \leqslant s^{\prime} .
$$

Поэтому из соотношения (5.7) в силу леммы 3 следует, что

$$
f(s)=0 \quad \forall s \leqslant s^{\prime}-\frac{f\left(s^{\prime}\right)}{1-\lambda},
$$

и, значит, $f(0)=0$, что противоречит сделанному допущению (5.5). Лемма 4 доказана.

ЛЕмма 5. Пусть в условиях и обозначениях леммы 4 функция $g(s)$ удовлетворяет дополнительно условию правильной монотонности:

$$
g(s-k g(s)) \geqslant\left(\varepsilon+k^{-1}\right) g(s) \quad \forall s<\infty .
$$

Тогда, в дополнение к утверждению леммы 4, справедлива следующая априорная оценка для $f(s)$ :

$$
f(s) \leqslant k g(s) \quad \forall s \geqslant 0 .
$$

ДокАЗАТЕЛЬСТво. Зафиксируем произвольное $s_{0}>s_{0}^{\prime}$ и определим последовательность $\left\{s_{j}\right\}$ :

$$
s_{j+1}=s_{j}-f\left(s_{j}\right), \quad j=0,1,2, \ldots
$$

В силу условия (5.3)

$$
f\left(s_{0}\right) \leqslant k g\left(s_{0}\right) .
$$

Предположим, что для некоторого $i \in \mathbb{N}$ мы уже установили, что

$$
f\left(s_{i}\right) \leqslant k g\left(s_{i}\right) .
$$

Тогда в силу соотношения (5.1), индуктивного предположения (5.10) и условия (5.8) имеем

$$
\begin{aligned}
f\left(s_{i+1}\right) & =f\left(s_{i}-f\left(s_{i}\right)\right) \leqslant \varepsilon f\left(s_{i}\right)+g\left(s_{i}\right)=\varepsilon f\left(s_{i}\right)+(\lambda-\varepsilon) f\left(s_{i}\right) \\
& =\lambda f\left(s_{i}\right) \leqslant \frac{\lambda}{\lambda-\varepsilon} g\left(s_{i}\right) \leqslant \frac{1}{\lambda-\varepsilon} g\left(s_{i}-\frac{g\left(s_{i}\right)}{\lambda-\varepsilon}\right) \\
& \leqslant(\lambda-\varepsilon)^{-1} g\left(s_{i}-f\left(s_{i}\right)\right)=k g\left(s_{i+1}\right), \quad \lambda=\varepsilon+k^{-1}<1 .
\end{aligned}
$$


Тем самым доказано, что

$$
f\left(s_{j}\right) \leqslant k g\left(s_{j}\right) \quad \forall j \in \mathbb{N} .
$$

Из определения последовательности $\left\{s_{i}\right\}$ следует

$$
s_{j+1} \geqslant s_{j}-k g\left(s_{j}\right) \geqslant s_{j}-\frac{a s_{j}}{\lambda-\varepsilon} \geqslant \lambda s_{j}>0 \quad \forall j \in \mathbb{N} .
$$

Поэтому $s_{j} \rightarrow \tilde{s} \geqslant 0$ при $j \rightarrow \infty$. В силу непрерывности функций $f(s), g(s)$ имеем

$$
f(\tilde{s})=g(\tilde{s})=0
$$

Из (5.11), (5.12) в силу произвольности выбора стартовой точки $s_{0}>s_{0}^{\prime}$ и непрерывности функций $f(s), g(s)$ следует доказьваемое соотношение (5.9). Лемма 5 доказана.

ЗАмЕчАнИЕ 5. Легко проверить, что функция $g(s)=a s$, где для параметра $a$ выполняется ограничение (5.4), удовлетворяет условию (5.8).

\section{Список литературы}

1. Мартинсон Л.К., Павлов К.Б. К вопросу пространственной локализации тепловых возмущений в теории нелинейной теплопроводности // ЖВМиМФ. 1972. Т. 12. C. 261-268.

2. Калашников A.C. Распространение возмущений в нелинейных средах при наличии поглощения // ЖВМиМФ. 1974. Т. 14. С. 70-85.

3. Калашников А. С. О дифференциальных свойствах обобщенных решений уравнений типа нестационарной фильтрации // Вестн. МГУ. 1974. Т. 29. С. 48-53.

4. Diaz J.I., Herrero M.A. Estimates on the support of the solutions of some nonlinear elliptic and parabolic problems // Proc.Roy.Soc.Edinburgh. 1981. V. 89A. P. 249-258.

5. Bertsch M., Nambu T., Peletier L. A. Decay of solutions of a degenerate nonlinear diffusion equations // Nonlinear. Anal. 1982. V. 6. P. 539-554.

6. Кершнер P. О поведении температурных фронтов в средах с нелинейной теплопроводностью при наличии поглощения // Вестн. МГУ. Сер. 1. Математика и механика. 1978. № 5. C. $44-51$.

7. Kersner $R$. Nonlinear heat conduction with absorption: space localization and extinction in finite time // SIAM J.Appl.Math. 1983. V. 43. P. 1274-1285.

8. Knops R. J., Straughan B. Decay and nonexistence for sublinearly forced systems in Continuum Mechanics // Nonlinear partial differ. equat. and their apll. College de France Seminar. V. II; // Research Notes in Mathematics 60 / Eds. H. Brezis and J.L. Lions. London: Pitmann, 1982. P. 251-264.

9. Bandle C., Stakgold I. The formation of the dead core in parabolic reaction-diffusion problems // Trans. Amer. Math. Soc. 1984. V. 286. P. 275-293.

10. Kamin S., Peletier L. A. Large time behaviour of solutions of the porous media equations with absorption. Report 14. Mathematical Institute. University of Leiden, 1985.

11. Diaz J.I., Hernandez J. Qualitative properties of free boundaries for some nonlinear degenerate parabolic equations // Nonlinear parabolic equations. Horlow: Longman, 1987. P. 85-93.

12. Friedman A., Herrero $M$. A. Extinction properties of semilinear heat equations with strong absorption // J. Math. Anal. Appl. 1987. V. 124. P. 530-546.

13. Chen Xu-Yan, Matano H., Mimura M. Finite point extinction and continuity of interfaces in nonlinear diffusion equation with strong absorption // J. Reine and Angew. Math. 1995. V. 459. № 1. P. 1-36.

14. Антонцев C. Н. О локализации решений нелинейных вырождающихся эллиптических и параболических уравнений // ДАН СССР. 1981. Т. 260. №6. С. 1289-1293.

15. Diaz J. I., Veron L. Local vanishing properties of solutions of elliptic and parabolic equations // Trans. Amer. Math. Soc. 1985. V. 290. № 2. P. 787-814. 
16. Antontsev S. N., Diaz J. I., Shmarev S. I. New results of the character of localization of solutions of elliptic and parabolic equations // International series of numerical mathematics. V. 106. Basel: Birkhauser, 1992. P. 59-66.

17. Антонцев C. Н., Шмарев C.И. Локальный энергетический метод и обращение в нуль слабых решений нелинейных параболических уравнений // ДАН СССР. 1991. Т. 318. № 4. C. $777-781$.

18. Antontsev S. N., Diaz J.I., Shmarev S.I. The support shrinking properties for local solutions of quasilinear parabolic equations with strong absorption term // Ann. Fac. Sciences de Toulouse. 1995. V. 4. № 1. P. 3-19.

19. Bernis $F$. Finite speed of propagation and asymptotic rates for some nonlinear higher order parabolic equations with absorption // Proc. of Royal Soc. Edinburgh. 1986. V. 104A. P. 1-19.

20. Шишков A.E. Об оценках скорости распространения возмущений в квазилинейных дивергентных вырождающихся параболических уравнениях произвольного порядка // Укр. матем. журн. 1992. Т. 44. № 10. С. 1451-1456.

21. Шишков A.E. Динамика геометрии носителя обобщенного решения квазилинейного дивергентного параболического уравнения высокого порядка // Дифференц. уравн. 1993. T. 29. №3. C. 537-547.

22. Lions J. L. Quelques methodes de resolution des problemes aux limites non lineaires. Paris: Dunod, 1969.

23. Bernis F. Existence results for doubly nonlinear higher order parabolic equations on unbounded domains // Math. Ann. 1988. V. 279. P. 373-394.

24. Nirenberg L. An extended interpolation inequality // Ann. Scuola Norm. Sup. Pisa. 1966. V. 20. P. 733-737.

Институт прикладной математики и механики НАН Украины, г. Донецк

Поступило в редакцию 15.III.1996 